\title{
Dynamics of fullerene coalescence
}

\author{
Yong-Hyun Kim, , 因 In-Ho Lee, ${ }^{2}$ K. J. Chang, ${ }^{1}$ and Sangsan Lee ${ }^{3}$ \\ ${ }^{1}$ Department of Physics, Korea Advanced Institute of Science and Technology, Daejeon 305-701, Korea \\ ${ }^{2}$ Korea Research Institute of Standards and Science, Daejeon 305-600, Korea \\ ${ }^{3}$ Korea Institute of Science and Technology Information, Daejeon 305-333, Korea
}

(Dated: October 30, 2018)

\begin{abstract}
Fullerene coalescence experimentally found in fullerene-embedded single-wall nanotubes under electron-beam irradiation or heat treatment is simulated by minimizing the classical action for many atom systems. The dynamical trajectory for forming a $(5,5) \mathrm{C}_{120}$ nanocapsule from two $\mathrm{C}_{60}$ fullerene molecules consists of thermal motions around potential basins and ten successive StoneWales-type bond rotations after the initial cage-opening process for which energy cost is about 8 $\mathrm{eV}$. Dynamical paths for forming large-diameter nanocapsules with $(10,0),(6,6)$, and $(12,0)$ chiral indexes have more bond rotations than 25 with the transition barriers in a range of $10-12 \mathrm{eV}$.
\end{abstract}

PACS numbers: 61.46.+w,34.10.+x,81.07.-b

Carbon nanostructures spanning from fullerenes 1] to single and multi-wall nanotubes [2], nano-peapods [3], and "Y" and "T" junctions [4] have attracted much attention from scientists for last two decades due to their unique structural, mechanical, and electrical properties 5]. In particular, carbon nanotubes and related junctions have great potential for applications on nanometric electronic devices such as quantum functional transistors and rectifiers since nanotubes can be semiconductors or metals depending upon their chiralities and diameters 6]. At the moment, however, development of the nanotubebased technology is limited because delicate control of their structures is not yet feasible.

Recently, merging processes between carbon nanostructures have been proposed as a new synthesis technique to construct more complicate functional structures: (1) Two nanotubes coalesce into diameter-doubled one in a high-energy $(1.25 \mathrm{MeV})$ electron beam condition 7 . (2) In the same condition, initially crossed nanotubes are found to form some functional junctions shaped like "Y" and "T" characters 4]. Since each carbon atom is displaced approximately every 100 sec by knock-on collisions with the high-energetic electrons, the irradiationinduced vacancies may play a crucial role in these merging processes. Terrones and his coworkers [4, 7] studied the vacancy-related merging mechanism utilizing the ordinary molecular dynamics technique. Though introducing various functional structures, this high-energy process may be not appropriate for nanometric device application because of the uncontrollable defect formation. (3) A low energy growth technique for nanotube bundles, where the chiral indexes of all individual nanotubes are almost perfectly mono-dispersed, was proposed in the mixture of $\mathrm{C}_{60}$ molecules and catalyst $\mathrm{Ni}$ pillars with a help of a $\sim 1.5 \mathrm{~T}$ magnetic field [8]. The annealing temperature is just $950{ }^{\circ} \mathrm{C}$. (4) In another low energy situation, a single-wall nanotube can be synthesized by merging fullerene molecules inside a nanotube vessel in nano-peapod systems [9, 10]. Of special importance is the last technique of fullerene coalescence because it is a catalyst-free and bottom-up process, having a potentiality for diameter and chirality control of single-wall nanotubes.

Nano-peapods [3] consist of array of fullerene molecules (inner peas) and a single-wall carbon nanotube (an outer pod), and all components are separated from the others by the van der Waals distance. The fullerene molecules as an one-dimensional crystal are spaced by about 0.97 $\mathrm{nm}$ or less, and the filling fraction is over $90 \%$. The high fraction is associated with the exothermic encapsulating process [12, 13]. Depending on preparation methods, the various fullerene species can be $\mathrm{C}_{60}, \mathrm{C}_{70}, \mathrm{C}_{82}$, and metallofullerenes [3, 9, 10, 11.

Electron-beam irradiation [9] or heat treatment 10] on these carbon composites induces coalescence of core fullerenes into an inside single-wall nanotube, of which diameter is $0.71 \mathrm{~nm}$ smaller than that of the outer 'vessel' tube. Since the electron beam in this case is irradiated for hundreds of seconds and its energy is about $0.1 \mathrm{MeV}$, we can not expect the irradiation-induced vacancies as like Refs. 4 and 7. Actually, the outer wall was little damaged during the coalescence process by the electron beams 9 ]. Iijima and his coworkers baked nano-peapods up to 1200 ${ }^{\circ} \mathrm{C}$ for 14 hours. They found that the inside fullerenes start to coalesce at $800{ }^{\circ} \mathrm{C}$ and complete transforming to a single-wall nanotube at $1200{ }^{\circ} \mathrm{C}$. They also proposed that diameters of the synthesized nanotubes are varying from 0.6 to $0.9 \mathrm{~nm}$ depending on the size of the outer 'vessel' tubes in a range of 1.3-1.6 nm [10]. This low energy merging process of fullerenes have great implication on diameter and chirality control in nanotube growths as we mentioned above, but little was known about its dynamical process. Furthermore, the ordinary molecular dynamics study for the process is not applicable because it never happens within the simulation time in use of the modern supercomputers.

Instead, topological consideration was made by Ueno et al. to the case of a $\mathrm{C}_{120}$ capsule with $(5,5)$ chi- 
ral index from two dimerized $\mathrm{C}_{60}$ fullerenes 14 ]. They found 22 successive Stone-Wales-type bond rotations as a topologically acceptable pathway for the transformation. Recently, hypothetic transition states between two consecutive local minima of the pathway was examined [15]. Similar topological analysis for head-to-head coalescence of capped nanotubes and fullerenes was made [16]: $(5,5)+(5,5),(10,10)+(10,10)$, and $\mathrm{C}_{60}+(10,10)$. However, these do not guarantee real dynamics of the coalescing process beyond topology. Fullerene collision [17, 18] and nanotube collision [19] were dealt with molecular dynamics techniques with the assumption of one constituent's high energetic incidence on the other. Fullerenes packed well inside single-wall nanotubes (nano-peapods) are not relevant with such a high energetic incidence.

In this Letter, to simulate the low-energy merging process of fullerene molecules with appropriate molecular dynamics information, we use the action-derived molecular dynamics method, recently proposed by Passerone and Parrinello 20] with some modifications for many atom systems [21].

The discretized version 22] of the classical action $(S)$ for numerical simulations is given by

$$
S=\sum_{j=0}^{P-1} \Delta\left(K_{j}-V_{j}\right),
$$

where $P$ is the total number of steps for discretization of simulation time $\tau, \Delta=\tau / P$ is the constant time interval, and $K_{j}\left(V_{j}\right)$ are the kinetic (potential) energy of the configuration $\mathbf{q}_{j}$ at the $j$-th time step. The instantaneous velocity is approximated as $\mathbf{v}_{j}=\left(\mathbf{q}_{j}-\mathbf{q}_{j+1}\right) / \Delta$, and then $K_{j}=m \mathbf{v}_{j}^{2} / 2$ where $m$ is the mass of each particle. Dynamical paths can be obtained by finding the stationary point of the action, i.e., $\delta S=0$, but in general it is a very difficult task to find the stationary point because it involves a root-finding problem which requires the second derivative of the potential. Furthermore, the action is not bounded and the stationary point can be either a local minimum/maximum, or a saddle.

To obtain effectively a dynamical path that is globally close to the Verlet trajectory, i.e., an optimal solution for the discretized action, we employ a rather simple minimization technique, where the action is supplemented with some penalty functions [20, 21];

$$
\begin{aligned}
& \tilde{\Theta}\left(\mathbf{q}_{j}, E ; K\right)=S+\mu_{E} \sum_{j=0}^{P-1}\left(E_{j}-E\right)^{2} \\
& \quad+\mu_{\mathbf{P}} \sum_{j=0}^{P-1} \mathbf{P}_{j}{ }^{2}+\mu_{\mathbf{L}} \sum_{j=0}^{P-1} \mathbf{L}_{j}{ }^{2}+\mu_{K}\left(\left\langle K_{j}\right\rangle-K\right)^{2} .
\end{aligned}
$$

Here, $\mathbf{P}_{j}\left(\mathbf{L}_{j}\right)$ is the instantaneous total linear (angular) momentum, and $E_{j}=K_{j}+V_{j}$ is the instantaneous total energy of $N$ particles at step index $j . E$ and $K$ is
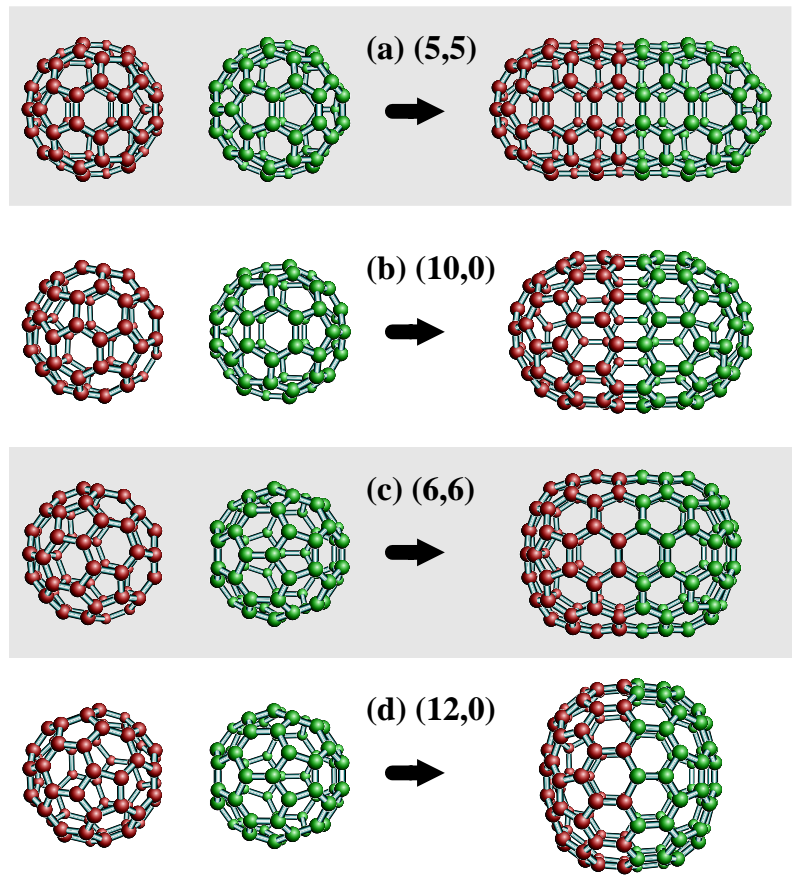

FIG. 1: (color online) Initial and final states for forming various $\mathrm{C}_{120}$ nanocapsules as precursors for fullerene-based nanotube growth.

the target total and kinetic energy, respectively, and all the $\mu$ 's are numerical parameters that enforce the corresponding constraints. The $\langle\cdots\rangle$ means the time average. With $\mu_{E}, \mu_{\mathbf{P}}$, and $\mu_{\mathbf{L}}$, we enforce the conservation laws of total energy, and total linear/angular momenta on the optimized trajectory. The last term in Eq. (2) is associated with the virial theorem that classical trajectories should satisfy, causing thermal motions around potential basins; in thermodynamic language, the virial theorem corresponds to the equipartition theorem.

In our action-derived molecular dynamics simulations, we minimize the generalized action $\tilde{\Theta}$ with respect to various atomic paths using a conjugate gradient technique for the given initial and final $N$-atom configurations, as shown in Fig. 1. The potential energy and Hellmann-Feynman forces are calculated using a tightbinding model for carbon systems 23]. Details of formulation and numerical procedure will be presented elsewhere [21].

For the fullerene coalescing process, we can reasonably assume that its initial state is two $\mathrm{C}_{60}$ molecules separated by $\sim 1 \mathrm{~nm}[9,10]$. However, for the final configuration, we can expect various $\mathrm{C}_{120}$ nanocapsules with different diameters and chiralities because experimentally the diameters of the synthesized inner tubes ranges in $0.6-0.9$ $\mathrm{nm}$ [10]. The simplest case is the $\mathrm{C}_{120}$ nanocapsule with diameter of $0.7 \mathrm{~nm}$ and chiral index of $(5,5)$ [Hereafter we call it the $(5,5)$ nanocapsule], as other workers considered [14, 15, 16]. We also present possible diameter 

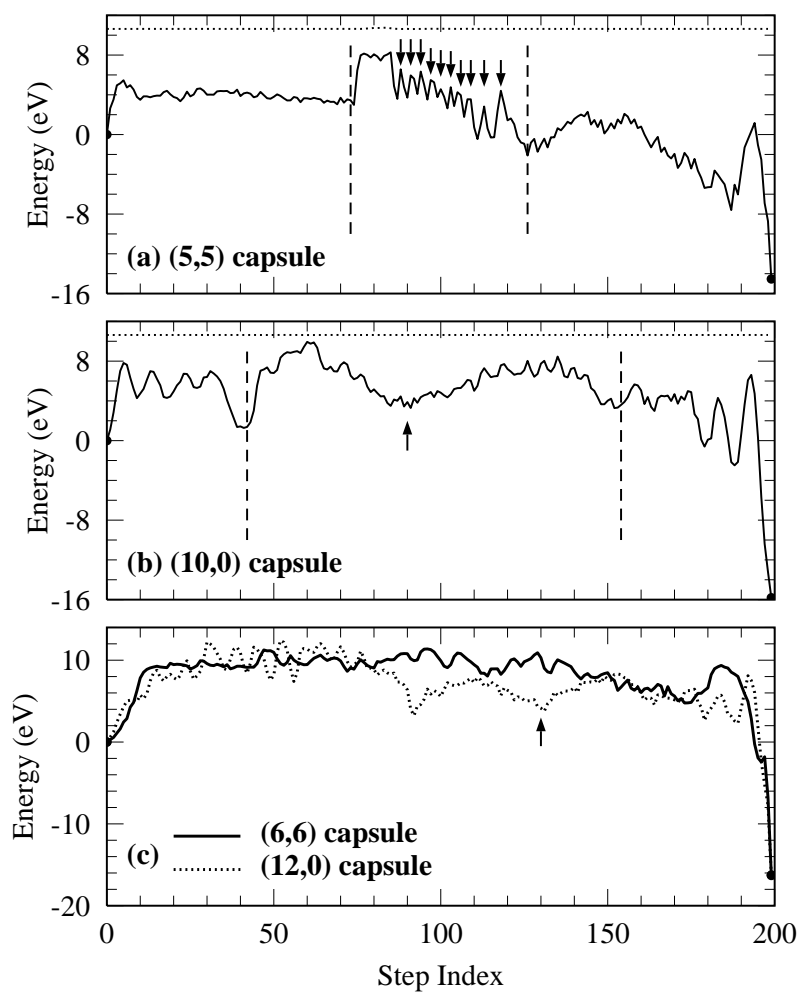

FIG. 2: Potential energy variation along the obtained dynamical trajectories of fullerene coalescence for forming various $\mathrm{C}_{120}$ nanocapsules. In (a) and (b), the solid (dotted) lines indicate the potential (total) energy of the $(5,5)$ and $(10,0)$ capsules, respectively, along the time step. We divide the trajectories into three parts as reactant, activation, and product regions in series. In (a), ten small arrows correspond to ten Stone-Wales-type bond rotations. In (b), the single arrow indicates when the intermediate $(5,5)$ capsule arises. In (c), we depict the potential energy variation in case of the $(6,6)$ and $(12,0) \mathrm{C}_{120}$ capsules. Also, the small arrow indicates forming the intermediate $(6,6)$ capsule in the trajectory of the $(12,0)$ capsule.

thickening process by forming $(10,0),(6,6)$, and $(12,0)$ nanocapsules with diameters of $0.8-9.8 \mathrm{~nm}$, as shown in Fig. 1. Assuming that all the atoms move least distance from initial to final states in order to accelerate the numerical convergence, we choose the initial orientation of two fullerene molecules differently depending on the cap structure of the final nanocapsules. With this we can remove free rotational motion of a single molecule in the full molecular dynamics simulation, with saving much computation time.

We perform action-derived molecular dynamics simulations using the parameters of $\mu_{E}=10^{8}, \mu_{\mathbf{P}}=\mu_{\mathbf{L}}=\mu_{K}=10^{4}$, $P=199$, and $\tau=P \Delta=1.2 \mathrm{ps}$, and display the results in Fig. 2. With the numerical parameters, we find that the total energy is well conserved over all time steps for all the nanocapsules, whereas the linear and angular momenta is well converged for the $(5,5)$ and $(10,0)$ capsules, but less strictly for $(6,6)$ and $(12,0)$ capsules. Since $E$ and $K$ cannot be independent variable in a microcanonical problem where total energy of systems is conserved, we relax $K$ within the minimization technique, regarding it as an extra variable after achieving some convergence.

For the coalescing process of the two $\mathrm{C}_{60}$ molecules into the $(5,5) \mathrm{C}_{120}$ nanocapsule, we can divide the obtained dynamical trajectory [See the supplementary movie] into three parts as reactant, activation, and product regions in series, as in Fig. 2(a). In the reactant region around the initial configuration, the 120 atoms of the two $\mathrm{C}_{60}$ molecules are randomly vibrated around their energy minima with preserving fullerene cage structures. This motion can be regarded as thermal motion, and be consistent with random trajectories around potential minima of the Mueller potential in Ref. 20. The relation $\left\langle K_{j}\right\rangle \approx\left\langle\triangle V_{j}\right\rangle$, the virial theorem for the harmonic potential, is satisfied. In the product region near the final state, we similarly find a thermal vibrating motion of the $(5,5)$ $\mathrm{C}_{120}$ nanocapsule. The coalescence occurs in-between, i.e., the activation region of about $0.3 \mathrm{ps}$. At the transition state, two fullerenes start to merge with breaking some $\mathrm{C}-\mathrm{C}$ bonds to open cage, and then a peanut-shaped intermediate structure appears at step index 87. Then, we can see 10 successive Stone-Wales-type bond rotations forming small potential peaks due to the occurrence of undercoordinated carbon atoms. The ten rotations are attributed to the 5-fold symmetry of the initial and final states, as discussed in Ref. 16. The peak height is about $2-5 \mathrm{eV}$. For the whole coalescence process, the energy barrier is estimated to $8.3 \mathrm{eV}$. This is quite comparable to the energy barrier for single Stone-Wales transformation in a $\mathrm{C}_{60}$ molecule 24].

For the $(10,0)$ nanocapsule, we also find the reactant and product regions of the dynamical trajectory with randomly vibrating atomic motion around both the potential basins. We count about 25 Stone-Walestype bond rotations in-between activation region, lasting about $0.6 \mathrm{ps}$ to complete the transformation. An intermediate $(5,5) \mathrm{C}_{120}$ nanocapsule is found at step index 90 on the trajectory for the $(10,0)$ capsule, indicated by the arrow in Fig. 2(b). Thus, we find a transformation route: two $\mathrm{C}_{60}$ fullerenes $\rightarrow(5,5) \mathrm{C}_{120}$ nanocapsule $\rightarrow$ $(10,0) \mathrm{C}_{120}$ nanocapsule. In the former part, a transition state occurs with an energy barrier of $9.9 \mathrm{eV}$, taking about 10 bond rotations, and in the latter part the $(5,5)$ nanocapsule transforms into $(10,0)$ nanocapsule through about 15 bond rotations, with an energy barrier of less than $8 \mathrm{eV}$. Even though the former process is conceptually same to the coalescence process for forming $(5,5)$ nanocapsule of Fig. 2(a), the energy barrier is higher by $1.6 \mathrm{eV}$ because of difference in the initial molecular orientation as shown in Figs. 1(a) and (b).

In the cases of $(6,6)$ and $(12,0)$ nanocapsules, the whole processes require more bond rotations than 30 . With the simulation time of $1.2 \mathrm{ps}$, the obtained dynamical trajec- 
TABLE I: Fullerene coalescence into various $\mathrm{C}_{120}$ nanocapsules. Diameter of capsules $(D)$, formation energy $\left(H_{f}\right)$, overall potential energy barriers $\left(V_{b}\right)$, average kinetic energy in Kelvin $\left(\left\langle K_{j}\right\rangle\right)$, and $O$ values are compared for the used target energy $(E)$.

\begin{tabular}{ccccccc}
\hline \hline & $D(\AA)$ & $H_{f}(\mathrm{eV})$ & $V_{b}(\mathrm{eV})$ & $\left\langle K_{j}\right\rangle(\mathrm{K})$ & $O($ a.u. $)$ & $E$ (a.u. $)$ \\
\hline$(5,5)$ & 6.85 & -14.53 & 8.3 & 556 & 599.5 & -29.85 \\
$(10,0)$ & 8.02 & -15.81 & 9.9 & 360 & 368.9 & -29.85 \\
$(6,6)$ & 8.32 & -16.25 & 11.4 & 239 & 227.7 & -29.80 \\
$(12,0)$ & 9.78 & -16.30 & 12.4 & 353 & 300.7 & -29.78 \\
\hline \hline
\end{tabular}

tories shown in Fig. 2(c) entirely belong to the activation process, different from those for the $(5,5)$ and $(10,0)$ nanocapsules in Fig. 2(a) and (b). If we use sufficiently large $\tau$ to cover 30-60 bond rotations, we may get such thermal vibrating modes near the initial and final states, but this demands much computational cost. With the limited simulation time, even though we can not guaranteed the real dynamics [In fact, total linear/angular momenta are poorly conserved], we can estimate satisfactorily activation energy barriers within the actionderived molecular dynamics formalism. The calculated values are 11.4 (step index 97) and $12.4 \mathrm{eV}$ (step index $50)$ for the $(6,6)$ and $(12,0)$ nanocapsules, respectively, rather high than those for the $(5,5)$ and $(10,0)$ nanocapsules. The limited simulation time causes the simultaneous Stone-Wales-type bond rotations, resulting the high energy barriers. Another reason is that, since we preserve the 6-fold symmetry in the initial and final states of these systems, double bonds of the hexagons should be broken to coalesce initially. This initial opening process needs an energy cost of about $10 \mathrm{eV}$, which corresponds to step index $0-30$. We also find an intermediate $(6,6)$ nanocapsule at step index 130 in the path of forming the $(12,0)$ nanocapsule, indicated by the arrow in Fig. 2(c).

We summarize all results and the target energy $(E)$ used for these four nanocapsules in Table I. As the diameters increase, the capsules are more stable with relaxing strain energy, but estimated energy barriers are higher. The large energy barriers over $10 \mathrm{eV}$ in the cases of the $(6,6)$ and $(12,0)$ nanocapsules are attributed to the limited simulation time and thus simultaneous bond rotations. Our estimated values for transition barriers are quite reasonable for low-energy merging process of fullerenes inside single-wall nanotubes. The value of 10 $\mathrm{eV}$ corresponds to system temperature of $1450 \mathrm{~K}$ for two $\mathrm{C}_{60}$ molecules, and the experimental temperature for the merging process is $1500 \mathrm{~K}[10]$. We also display the average kinetic energy $\left\langle K_{j}\right\rangle$ used for ensuring the virial theorem, and the discretized Onsager-Machlup actions $O$ 25]. The obtained $O$ values are rather large, especially for the cases of the $(5,5)$ nanocapsule due to the thermal motions in dynamical trajectory. We note that the action-derived molecular dynamics cannot offer a unique solution for the dynamical paths of a specific rare event. We may obtain different dynamical paths with various $O$ and $\left\langle K_{j}\right\rangle$, depending on the target energy, initial seed, and minimization schedule for the functional Eq. (2). Nevertheless, we believe that the $\tilde{\Theta}$ trajectory generally reflects the real dynamics of the system under consideration via the constraints for the classical laws. By imposing the appropriate dynamical laws and virial theorem, we can obtain an estimate, not exact value, for transition energy barrier and transformation process for specific rare events based on the real dynamics.

In conclusion, we investigate the molecular transformation of two $\mathrm{C}_{60}$ fullerenes into various $\mathrm{C}_{120}$ nanocapsules, and find dynamical paths for the fullerene coalescence using the action-derived molecular dynamics technique. In the dynamical process of forming the various $\mathrm{C}_{120}$ nanocapsule with the chiral indexes of $(5,5),(10,0)$, $(6,6)$, and $(12,0)$, we find that the process consists of successive Stone-Wales-type bond rotations with the transition energy barrier of about 8-12 eV. Further growth via coalescence between nanocapsules and $\mathrm{C}_{60}$ fullerenes may be natural extension of the above coalescence process with great similarity, requiring future study.

The authors thank T.-W. Ko. for useful discussion. This work is supported by the GRID project of KISTI.

* yonghyun@mail.kaist.ac.kr

[1] H.W. Kroto et al., Nature (London) 318, 162 (1985).

[2] S. Iijima, Nature (London) 354, 56 (1991).

[3] B.W. Smith et al., Nature (London) 396, 323 (1998).

[4] M. Terrones et al., Phys. Rev. Lett. 89, 075505 (2002).

[5] M.S. Dresselhaus et al., Science of Fullerenes and Carbon Nanotubes (Academic, San Diego, 1996).

[6] C. Dekker, Phys. Today 52 (5), 22 (1999).

[7] M. Terrones et al., Science 288, 1226 (2000).

[8] R.R. Schlittler et al., Science 292, 1136 (2001).

[9] D.E. Luzzi and B.W. Smith, Carbon 38, 1751 (2000).

[10] S. Bandow et al., Chem. Phys. Lett. 337, 48 (2001).

[11] K. Hirahara et al., Phys. Rev. Lett. 85, 5384 (2000).

[12] S. Okada et al., Phys. Rev. Lett. 86, 3835 (2001).

[13] S. Berber et al., Phys. Rev. Lett. 88, 185502 (2002).

[14] H. Ueno et al., Fullerene Sci. Techn. 6, 319 (1998).

[15] M. Yoon et al. (to be published).

[16] Y. Zhao et al., Phys. Rev. Lett. 88, 185501 (2002).

[17] B.L. Zhang et al., J. Phys. Chem. B 97, 3134 (1993).

[18] Y. Xia et al., Phys. Rev. B 53, 13871 (1996).

[19] T. Kawai et al., Phys. Rev. Lett. 89, 085901 (2002).

[20] D. Passerone and M. Parrinello, Phys. Rev. Lett. 87, 108302 (2001).

[21] Y.-H. Kim et al. (to be published).

[22] R.E. Gillilan and K.R. Wilson, J. Chem. Phys. 97, 1757 (1992).

[23] C.H. Xu et al., J. Phys: Cond. Matt. 4, 6047 (1992).

[24] J.-Y. Yi and J. Bernholc, J. Chem. Phys. 96, 8634 (1992).

[25] R. Olender and R. Elber, J. Chem. Phys. 105, 9299 (1996). 